\title{
Efeitos da Suplementação de Creatina Sobre Força e Hipertrofia Muscular: Atualizações
}

\author{
Effects of Creatine Supplementation on Strength and Muscle \\ Hypertrophy: Current Concepts
}

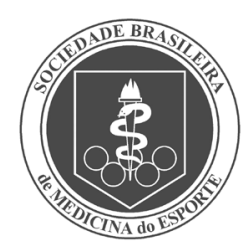

Artigo de Revisão

\author{
Bruno Gualano, 14 \\ Fernanda Michelone Acquesta ${ }^{2}$ \\ Carlos Ugrinowitsch ${ }^{3}$ \\ Valmor Tricoli ${ }^{3}$ \\ Júlio Cerca Serrão \\ Antonio Herbert Lancha Junior?

\begin{abstract}
1. Laboratório de Nutrição e Metabolismo Aplicados à Atividade Motora. Escola de Educação Física e Esporte. Departamento de 2. Laboratório de Biomecânica. Escola de Educação Física e Esporte. Departamento de Biodinâmica do Movimento Humano.

3. Laboratório de Adaptações Neuromusculares ao Treinamento de Força. Escola de Educação Física e Esporte. Departamento de Esporte. 4. Laboratório de Avaliação e Condicionamento em Reumatologia. Hospital das Clínicas, Faculdade de Medicina.
\end{abstract} \\ Biodinâmica do Movimento Humano.
}

Endereço para correspondência: Avenida Professor Mello Moraes, 65, Butantã - 05508-030 - São Paulo, SP. Brasil.

E-mail: gualano@usp.br

\begin{abstract}
RESUMO
A suplementação de creatina vem sendo utilizada amplamente na tentativa de aumentar força e massa magra em sujeitos saudáveis e atletas. Além disso, diversos estudos têm sido conduzidos no intuito de desvendar os mecanismos responsáveis pelas eventuais adaptações a esse suplemento. Diante disso, essa revisão teve como objetivos: 1) discutir os principais estudos que investigaram os efeitos da suplementação de creatina na força e hipertrofia; e 2) reunir as evidências acerca dos possíveis mecanismos responsáveis pelo aumento de força e massa magra como consequência desse suplemento, enfatizando os mais recentes achados e as perspectivas sobre o tema. De fato, existem fortes evidências demonstrando que a suplementação de creatina é capaz de promover aumentos de força e hipertrofia. Os efeitos desse suplemento sobre a retenção hídrica, o balanço proteico, a expressão de genes/proteínas associados à hipertrofia e ativação de células satélites, podem explicar as adaptações musculoesqueléticas observadas. Diante desses achados, os potenciais efeitos terapêuticos desse suplemento emergem como um futuro e promissor campo de estudo.
\end{abstract}

Palavras-chave: efeitos ergogênicos, mecanismos, suplementos nutricionais.

\begin{abstract}
Creatine supplementation has been widely used to increase both muscle strength and lean mass in healthy individuals and athletes. Furthermore, several studies have investigated the mechanisms responsible for such adaptations. Thus, this review aimed at 1) examining the major studies investigating the effects of creatine supplementation on strength and hypertrophy, and 2) exploring the mechanisms underlying these responses, stressing the most recent findings and perspectives regarding creatine supplementation. There is strong evidence demonstrating that creatine supplementation is able to enhance strength and hypertrophy. The effects of creatine on water retention, protein balance, genes/proteins related to hypertrophy, and satellite cells activation may explain the creatine-mediated muscle skeletal adaptations. In light of these findings, the potential therapeutic effects of creatine supplementation might be considered as a promising clinical and research field.
\end{abstract}

Keywords: ergogenic effects, mechanisms, nutritional supplements.

\section{INTRODUÇÃO}

A creatina (ácido a-metil guanidino acético) é uma amina de ocorrência natural sintetizada endogenamente pelo fígado, rins e pâncreas, a partir dos aminoácidos glicina e arginina (1). Pode também ser obtida via alimentação, especialmente pelo consumo de carne vermelha e peixes. A produção endógena (1 $\mathrm{g} / \mathrm{dia})$ somada à obtida na dieta (1 g/dia para uma dieta onívora) se iguala à taxa de degradação espontânea da creatina e fosfocreatina sob a forma de creatinina, por reação não enzimática ${ }^{(1)}$. A creatina é encontrada no corpo humano nas formas livre (60 a 70\%) e fosforilada (30 a 40\%). Cerca de $95 \%$ é armazenada no músculo esquelético, sendo que o restante situa-se no coração, músculos lisos, cérebro e testículos(2).

Desde que foi demonstrado que a suplementação de creatina (20g/dia por cinco-sete dias) promove aumento de 20\% nas concentrações de creatina muscular ${ }^{(3)}$, diversos estudos passaram a investigar o efeito dessa suplementação no rendimento físico-esportivo ${ }^{(4-9)}$. Os efeitos ergogênicos da suplementação de creatina em atividades intermitentes, como o treinamento de força, são bem descritos (para detalhes, ver Terjung et al.(2)). De fato, diversos estudos, incluindo duas meta-análises, demonstram que a suplementação de creatina pode promover ganhos de força e massa magra ${ }^{(10-17)}$.

Embora os efeitos ergogênicos da suplementação de creatina no treinamento de força sejam bem documentados, os mecanismos pelos quais essas adaptações ocorrem não são totalmente elucidados. A origem do ganho de massa magra, por exemplo, tem sido alvo de grandes discussões, já que é incerto se o fator responsável por essa adaptação se refere meramente a uma retenção hídrica ou a uma "verdadeira" hipertrofia. Recentes achados têm indicado que a suplementação de creatina pode alterar a transcrição de fatores miogênicos regulatórios ${ }^{(16-21)}$, aumentar a eficiência de tradução proteica através da via hipertrófica PI3K-AKT/PKB-mTOR ${ }^{(22)}$ e controlar a ativação, proliferação e diferenciação de células satélites ${ }^{(23)}$. Contudo, pesquisadores ainda divergem se a creatina per se é capaz de promover tais efeitos ou se a combinação ao treinamento de força é necessária.

Em 2003, a Sociedade Brasileira de Medicina do Esporte publicou a inédita e, até hoje, única diretriz nacional sobre suplementos alimentares ${ }^{(24)}$. Os autores não recomendaram o uso da suplementação de creatina à população em geral, mesmo em atletas, considerando que, à época, evidências científicas que corroborassem o emprego desse suplemento eram escassas. Diante disso, faz-se necessária uma atualização sobre o tema, tendo essa revisão os seguintes objetivos: I) descrever os principais estudos que investigaram os efeitos da suplementação de creatina na produção de força muscular; II) descrever os efeitos da combinação treinamento de força e suplementação de creatina na hipertrofia muscular; III) descrever os possíveis mecanismos 
responsáveis pelo aumento de força e massa magra como consequência desse suplemento, enfatizando os mais recentes achados e as perspectivas sobre o tema.

Para tanto, foram buscados estudos na base de dados Medline, no período de 1992 a 2009, utilizando-se as palavras-chave: creatine supplementation "and" power, strength, hypertrophy, muscle mass, lean mass. Os estudos que traziam informações originais e relevantes à literatura foram inclusos no presente trabalho. Além disso, revisões sistemáticas e narrativas que contribuíam para o entendimento do tema também foram consideradas.

\section{Efeitos da suplementação de creatina na produção de força muscular}

A análise da literatura indica o potencial efeito ergogênico da suplementação de creatina na capacidade do músculo gerar força, corroborada por duas meta-análises ${ }^{(10,11)}$. Em contrapartida, há controvérsias acerca da capacidade da creatina per se em proporcionar essa adaptação.

Existem indícios, no entanto, que mesmo na ausência de treinamento de força, a suplementação de creatina poderia ter um efeito benéfico na força muscular, mediado por diversos mecanismos, tais como: I) aumento dos conteúdos intramusculares de fosforilcreatina; II) aumento da velocidade de regeneração de fosforilcreatina durante o exercício; III) melhora na atividade da via glicolítica pelo tamponamento de íons $\mathrm{H}^{+}$; IV) diminuição do tempo de relaxamento no processo contração-relaxamento da musculatura esquelética, em decorrência da melhora na atividade da bomba sarcoendoplasmática de cálcio; e V) aumento da concentração de glicogênio muscular ${ }^{(2,7,25-27)}$. Comparados aos demais, os dois primeiros fatores são aqueles que, teoricamente, mais explicariam a melhora aguda de desempenho(2).

Contudo, ressalta-se que nem todos os trabalhos verificaram melhora na força em consequência desse suplemento. Sabidamente, fatores como baixo poder estatístico, variabilidade individual na resposta à suplementação e falta de controle nutricional explicam parcialmente essa divergência na literatura. Essas variáveis que influenciam a resposta à suplementação de creatina são bastante exploradas nas revisões de Lemon ${ }^{(28)}$ e Gualano et al. ${ }^{(29)}$ e, portanto, não serão abordadas em profundidade nesse trabalho. Além disso, o leitor deve ter em mente que a magnitude dos ganhos de força observados em estudos com creatina pode ser superestimada em função de uma falha metodológica. Tarnopolsky et al. ${ }^{(30)}$ demonstraram que sujeitos suplementados com creatina e treinados por oito semanas não tiveram maior ganho de força quando comparados a seus pares submetidos ao mesmo tipo de treinamento mas suplementados com uma dieta isoenergética e isonitrogenada. Esses provocativos achados sugerem que parte do efeito ergogênico da creatina observado na grande maioria dos estudos pode ser creditado às diferenças na composição nitrogenada e energética dos suplementos de creatina vs. placebos.

Embora os estudos revelem, em sua maioria, o potencial ergogênico da creatina per se sobre a força, evidências sugerem que a combinação desse suplemento ao treinamento pode ser mais eficaz ${ }^{(31)}$.

Arciero et al. ${ }^{(32)}$ realizaram um interessante estudo com o intuito de quantificar as diferenças entre os efeitos diretos e indiretos (combinados ao treinamento de força) da suplementação de creatina. Os autores dividiram os sujeitos em dois grupos: apenas suplementados com creatina ou suplementados com creatina e submetidos a treinamento de força. Ao final de quatro semanas, foi verificado que o primeiro grupo apresentou aumentos de força dinâmica máxima no supino (8\%) e leg press (16\%), enquanto que o segundo grupo demonstrou aumento nessas mesmas variáveis de 18 e 42\%, respectivamente. Diante desses achados, Arciero et al. ${ }^{(32)}$ sugerem que cerca $40 \%$ do aumento de força no grupo treinado e suplementado se deva aos efeitos agudos da suplementação de creatina, sendo o restante devido a mecanismos mediados pelo treinamento. Embora seja uma estimativa indireta, já que se baseia em meros cálculos percentuais, esse estudo tem o mérito de ser o primeiro e, ao nosso conhecimento, o único a buscar diferenciar os efeitos per se da creatina daqueles combinados ao treinamento de força. A tabela 1 descreve os trabalhos que investigaram os efeitos da creatina, acompanhada ou não de treinamento, na capacidade de força.

\section{Efeitos da suplementação de creatina na hipertrofia}

Vários são os estudos que evidenciaram maiores aumentos na massa magra em consequência da suplementação de creatina, combinada com o treinamento de força(4,7,10-17,23,33). Em meta-análise conduzida por Branch ${ }^{(10)}$, dos 67 estudos que mensuraram a massa corporal, 43 reportaram aumentos na massa corporal total e/ou massa magra decorrentes da suplementação de creatina.

Existem evidências suficientes para se afirmar que a suplementação de creatina acompanhada de treinamento de força resulta em aumentos de hipertrofia maiores do que aqueles vistos quando da suplementação ou treinamento isoladamente. Volek et al.(13), por exemplo, evidenciaram que sujeitos que receberam suplementação de creatina durante treinamento de força de 12 semanas apresentaram maiores aumentos na área de secção transversa de fibras do tipo I, Ila e IIx em relação ao grupo controle, apenas treinado.

Contudo, os mecanismos fisiológicos que explicam esse maior aumento da musculatura com a suplementação de creatina juntamente com o treinamento de força ainda não foram esclarecidos, entretanto existem muitas hipóteses sendo investigadas. A seguir, discutiremos as recentes evidências sobre cada uma delas.

\section{Efeitos da creatina sobre a retenção hídrica e balanço proteico}

Talvez um dos primeiros achados fisiológicos atribuídos à suplementação de creatina tenha sido o aumento no volume total de água

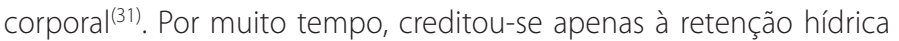
o notório ganho de massa magra e peso corporal decorrentes desse suplemento. Recentemente, contudo, tem sido especulado que mudanças nos conteúdos intracelulares de água possam influenciar a tradução de proteínas contráteis ${ }^{(19,23,31)}$. Berneis et al. ${ }^{(34)}$ foram os primeiros a fornecer importantes indícios que sustentam essa hipótese em humanos. Os autores submeteram homens saudáveis às três condições definidas como de hipo-osmolaridade, hiperosmolaridade e iso-osmolaridade extracelular e observaram que, no primeiro caso, houve maior aumento na síntese proteica do que nas outras situações. Esse achado foi atribuído ao "inchaço" celular promovido pelo meio extracelular hiperosmótico. Resta saber até que ponto a suplementação de creatina é capaz de alterar significativamente o balanço hídrico, a osmolaridade celular e, por consequência, o balanço proteico em humanos.

De fato, poucos estudos investigaram se a suplementação de creatina pode alterar o catabolismo ou a síntese de proteínas. Parise et al. ${ }^{(35)}$ demonstraram, através da técnica de infusão contínua de leucina marcada, que a suplementação de creatina durante cinco dias não afeta a síntese proteica, embora tenha reduzido o catabolismo proteico em homens. Louis et al. ${ }^{(36)}$ não observaram alterações na síntese e catabolismo de proteínas, após cinco dias de suplementação. O mesmo grupo falhou novamente em documentar possíveis alterações no balanço proteico quando da suplementação de creatina acompanhada de uma sessão de treinamento de força ${ }^{(37)}$. Esses dados sugerem que a creatina per se, por um curto período, não altera significativamente o balanço proteico, mesmo quando combinada a uma única sessão de exercícios de força. Estudos futuros devem avaliar se a suplementação de creatina afeta o balanço proteico em longo prazo.

\section{Efeitos da creatina sobre o volume de treinamento}

Alternativamente, considera-se que a suplementação de creatina parece ter grande efeito sobre o aumento no volume de treinamento, 
Tabela 1. Efeitos da suplementação de creatina sobre o desempenho de força e massa corporal.

\begin{tabular}{|c|c|c|c|c|}
\hline Autores e ano & Características da amostra & Regime de suplementação & Protocolo de treinamento & Principais resultados \\
\hline Arciero et al. $(2001)^{(32)}$ \# & $\begin{array}{l}30 \text { homens saudáveis } \\
\text { não treinados }\end{array}$ & $\begin{array}{l}20 \mathrm{~g} / \mathrm{d} \text { por } 5 \mathrm{~d}+ \\
10 \mathrm{~g} / \mathrm{d} \text { por } 4 \mathrm{sem}\end{array}$ & $\begin{array}{l}3 \mathrm{~d} / \mathrm{sem}, 2 \times 10 \text { rep (70\% de } 1 \mathrm{RM})+1 \mathrm{~s} \text { até } \\
\text { exaustão }\end{array}$ & $\begin{array}{l}\text { 个2,5\% massa corporal } \\
\uparrow 2,7 \% \text { massa magra } \\
\uparrow 17,8 \% 1 \mathrm{RM} \text { supino } \\
\uparrow 42,3 \% \text { 1RM leg press } \\
\end{array}$ \\
\hline Bemben et al. $(2001)^{(50)}$ & $\begin{array}{l}25 \text { jogadores de futebol } \\
\text { americano }\end{array}$ & $\begin{array}{l}20 \mathrm{~g} / \mathrm{d} \text { por } 5 \mathrm{~d}+ \\
5 \mathrm{~g} / \mathrm{d} \text { por } 9 \mathrm{sem}\end{array}$ & $\begin{array}{l}\text { 2d/sem, } 3 \text { a } 5 \times 8 \text { a } 12 \text { rep ( } 70 \% \text { a } 105 \% \text { de } 1 \mathrm{RM}) \\
+2 \mathrm{~d} / \mathrm{sem}, 3 \text { a } 5 \times 2 \text { a } 4 \text { rep ( } 65 \% \text { a } 95 \% \text { de } 1 \mathrm{RM})\end{array}$ & $\begin{array}{l}\uparrow 5,5 \% 1 \mathrm{RM} \text { supino } \\
\uparrow, 7 \% \text { 1RM agachamento } \\
\leftrightarrow \text { pico de torque do quadríceps } \\
\leftrightarrow \text { pico de torque dos isquiotibiais a } 60 \text { e } 180 \% \text { s } \\
\uparrow 17,3 \% \text { pico de torque dos isquiotibiais a } 300 \% \text { s }\end{array}$ \\
\hline Bermon et al. $(1998)^{(51)} \S$ & $\begin{array}{l}35 \text { homens e mulheres } \\
\text { idosos não treinados }\end{array}$ & $\begin{array}{l}20 \mathrm{~g} / \mathrm{d} \text { por } 5 \mathrm{~d}+ \\
3 \mathrm{~g} / \mathrm{d} \text { por } 5 \mathrm{sem}\end{array}$ & $3 \mathrm{~d} / \mathrm{sem}, 3 \times 8$ rep ( $80 \%$ de $1 \mathrm{RM})$ & \begin{tabular}{|l|}
$* \leftrightarrow$ massa corporal total \\
$* \uparrow 17,3 \% 1$ RM leg press $* \uparrow 15,8 \% 12 \mathrm{RM}$ leg press \\
$* \uparrow 17,4 \% 1$ RM supino \\
$* \uparrow 19,4 \% 12 \mathrm{RM}$ supino \\
$* \uparrow 18,5 \% 1$ RM extensão de joelho \\
$* \uparrow 16,3 \% 1$ RM extensão de joelho \\
\end{tabular} \\
\hline Burke et al. (2003) $)^{(52)}$ & $\begin{array}{l}43 \text { adultos saudáveis não } \\
\text { treinados, vegetarianos } \\
\text { ou não }\end{array}$ & $\begin{array}{l}0,25 \mathrm{~g} / \mathrm{kg} / \mathrm{d} \text { por } 7 \mathrm{~d}+ \\
0,0625 \mathrm{gg} / \mathrm{d} \text { por } 8 \mathrm{sem}\end{array}$ & $\begin{array}{l}3 \mathrm{~d} / \mathrm{sem} \\
\text { Sem } 1: 3 \times 10-12 \mathrm{RM} \\
\text { Sem } 2: 3 \times 8-10 \mathrm{RM} \\
\text { Sem } 3: 4 \times 6-8 \mathrm{RM} \\
\text { Sem } 4: 5 \times 4-6 \mathrm{RM} \\
\text { Sem 5: } 4 \times 6-8 \mathrm{RM} \\
\text { Sem } 6: 3 \times 8-10 \mathrm{RM} \\
\text { Sem } 7: 3 \times 10-12 \mathrm{RM}\end{array}$ & $\begin{array}{l}\text { T massa corporal total } \\
\uparrow 9 \% \text { massa magra (vegetarianos) } \\
\uparrow 4,3 \% \text { massa magra (não vegetarianos) } \\
\uparrow 1 \text { RM supino } \\
* \uparrow 1 \text { RM leg press }\end{array}$ \\
\hline Candow et al. $(2008)^{(53)}$ & $\begin{array}{l}40 \text { homens idosos saudáveis } \\
\text { não treinados }\end{array}$ & $\begin{array}{l}0,1 \mathrm{~g} / \mathrm{kg} / \mathrm{d} \text { nos dias de } \\
\text { treinamento }\end{array}$ & $3 \mathrm{~d} / \mathrm{sem}, 3 \times 10 \mathrm{rep}(70 \%$ 1RM) & 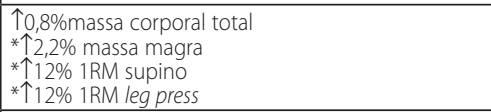 \\
\hline Chrusch et al. (2001) & $\begin{array}{l}30 \text { homens idosos } \\
\text { não treinados }\end{array}$ & $\begin{array}{l}0,3 \mathrm{~g} / \mathrm{kg} / \mathrm{d} \text { por } 5 \mathrm{~d}+0,07 \mathrm{~g} / \\
\mathrm{kg} \text { por } 12 \text { sem }\end{array}$ & $3 \mathrm{~d} / \mathrm{sem}, 3 \times 10 \mathrm{rep}$ & 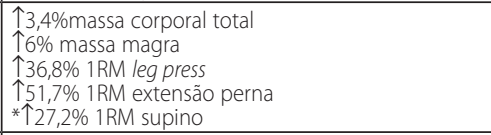 \\
\hline Cribb e Hayes (2006) ${ }^{(55)}$ & $\begin{array}{l}23 \text { adultos saudáveis } \\
\text { treinados }\end{array}$ & $\begin{array}{l}\text { 0,07g/kg/dia nos dias de } \\
\text { treinamento ( } 2 \text { grupos: } \\
\text { imediatamente antes e após } \\
\text { cada sessão ou em horários } \\
\text { espaçados) }\end{array}$ & $\begin{array}{l}\text { 4d/sem } \\
\text { 1a fase: 70-75\% 1RM } \\
\text { 2a fase: } 80-85 \% \text { RMM } \\
\text { 3a fase: } 90-95 \% \text { 1RM }\end{array}$ & $\begin{array}{l}\text { Beneficios do consumo imediatamente antes e após } \\
\text { suplementação: } \\
\uparrow 3 \% \text { massa corporal total } \\
\uparrow 4 \% \text { massa magra } \\
\uparrow 14,1 \% 1 \mathrm{RM} \text { agachamento } \\
\uparrow 9,6 \% 1 \mathrm{RM} \text { supino } \\
* \uparrow 12,3 \% \text { 1 RM levantamento terra }\end{array}$ \\
\hline Cribb et al. $(2007)^{(56)}$ & $\begin{array}{l}31 \text { adultos saudáveis } \\
\text { treinados }\end{array}$ & $0,1 \mathrm{~g} / \mathrm{kg} / \mathrm{d}$ por $10 \mathrm{sem}$ & $\begin{array}{l}\text { 4d/sem } \\
\text { Sem 1 e 2: 10RM } \\
\text { Sem 3 a 6: 6-8RM } \\
\text { Sem 7 a 10: 4-6RM }\end{array}$ & $\begin{array}{l}* \uparrow \uparrow, 9 \% \text { massa corporal total } \\
\uparrow 9,9 \% \text { massa magra } \\
\uparrow 28,4 \% \text { 1RM agachamento } \\
\uparrow 20,7 \% \text { 1 RM supino } \\
\uparrow 17,2 \% \text { 1 RM pulley } \\
\end{array}$ \\
\hline Cribb et al. $(2007)^{(57)}$ & $\begin{array}{l}33 \text { adultos saudáveis } \\
\text { treinados }\end{array}$ & $\begin{array}{l}0,3 \mathrm{~g} / \mathrm{kg} / \mathrm{d} \text { por } 1 \mathrm{sem}+ \\
0,1 \mathrm{~g} / \mathrm{kg} / \mathrm{d} \text { por } 10 \mathrm{sem}\end{array}$ & $\begin{array}{l}\text { 4d/sem } \\
\text { 1a fase: } 70-75 \% 1 \text { RM } \\
\text { 2a ase: } 80-85 \% \text { RMM } \\
\text { 3a fase: } 90-95 \% \text { RMM }\end{array}$ & $\begin{array}{l}* \uparrow 4,7 \% \text { massa corporal total } \\
\uparrow 6,4 \% \text { massa magra } \\
\uparrow 25 \% \text { 1RM agachamento } \\
\uparrow 19 \% \text { 1RM supino } \\
\uparrow 16,6 \% 1 \text { 1RM pulley } \\
\end{array}$ \\
\hline Hass et al. $(2007)^{(58)}$ & $\begin{array}{l}10 \text { homens e mulheres } \\
\text { idosos com doença de } \\
\text { Parkinson }\end{array}$ & $\begin{array}{l}20 \mathrm{~g} / \mathrm{d} \text { por } 5 \mathrm{~d}+5 \mathrm{~g} / \mathrm{d} \text { por } \\
12 \mathrm{sem}\end{array}$ & $2 \mathrm{~d} / \mathrm{sem}, 1 \times 8-12 \mathrm{RM}$ & 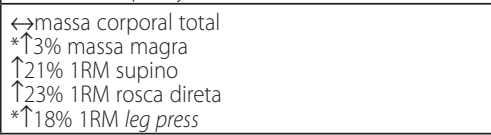 \\
\hline Hespel et al. $(2001)^{(33)}$ & $\begin{array}{l}22 \text { homens e mulheres } \\
\text { adultos saudáveis, } \\
\text { imobilizados por } 2 \text { sem. }\end{array}$ & $\begin{array}{l}20 \mathrm{~g} / \mathrm{d} \text { por } 2 \mathrm{sem} \\
\text { (imobilização) }+15 \mathrm{~g} / \mathrm{d} \text { por } 3 \\
\text { sem }+5 \mathrm{~g} / \mathrm{d} \text { por } 7 \mathrm{sem}\end{array}$ & $\begin{array}{l}\text { 3d/sem } \\
\text { Sem } 1 \text { a 3: } 4 \times 12 \text { extensões de joelho unilaterais } \\
\text { (60\%torque isométrico máximo) } \\
\text { Sem } 4 \text { a } 10: 6 \times 12 \\
\end{array}$ & $\begin{array}{l}\uparrow 21 \% \text { AST quadríceps } \\
\uparrow 52 \% \text { potência quadríceps } \\
* \uparrow 42 \% \text { torque máximo quadríceps }\end{array}$ \\
\hline Hoffman et al. (2006) ${ }^{(59)}$ & $\begin{array}{l}33 \text { jogadores de futebol } \\
\text { americano }\end{array}$ & 10,5g/d por $10 \mathrm{sem}$ & $\begin{array}{l}\text { 4d/sem } \\
\text { Sem } 1 \text { e } 2: 4 \times 8-10 \mathrm{RM} \\
\text { Sem } 3 \text { a } 6: 4 \times 6-8 \mathrm{RM} \\
\text { Sem } 7 \text { a } 10: 5 \times 4-6 \mathrm{RM} \\
\end{array}$ & $\begin{array}{l}\mid \leftrightarrow \text { massa corporal total } \\
\uparrow 13 \% \text { 1RM supino } \\
\uparrow 15 \% \text { 1RM agachamento }\end{array}$ \\
\hline Jowko et al. $(2001)^{(12)}$ & $\begin{array}{l}40 \text { adultos saudáveis } \\
\text { não treinados }\end{array}$ & $\begin{array}{l}20 \mathrm{~g} / \mathrm{d} \text { por } 7 \mathrm{~d}+10 \mathrm{~g} \text { por } \\
3 \mathrm{sem}\end{array}$ & $3 \mathrm{~d} / \mathrm{sem}, 3$ a $4 \times 6$ a 8 rep (60\% a $75 \%$ de 1 RM) & 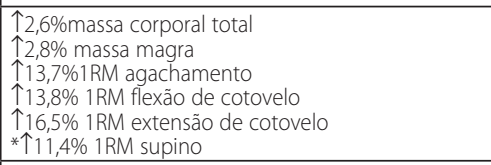 \\
\hline Kerksick et al. $(2007)^{(60)}$ & $\begin{array}{l}49 \text { homens e mulheres } \\
\text { adultos saudáveis treinados }\end{array}$ & $3 \mathrm{~g} / \mathrm{dia}$ por $12 \mathrm{sem}$ & $\begin{array}{l}\text { 4d/sem } \\
\text { Sem } 1 \text { a } 4: 3 \times 10 R M \\
\text { Sem } 5 \text { a } 8: 3 \times 8 R M \\
\text { Sem } 9 \text { a } 12: 3 \times 6 R M\end{array}$ & $\begin{array}{l}\uparrow 2,2 \% \text { massa corporal total } \\
\uparrow, 2 \% \text { massa magra } \\
* \uparrow 7,3 \% 1 \text { RM supino } \\
* \uparrow 11,5 \% 1 \text { RM leg press } \\
*\end{array}$ \\
\hline Kreider et al. $(1998)^{(61)}$ & $\begin{array}{l}25 \text { jogadores de futebol } \\
\text { americano }\end{array}$ & $15,75 \mathrm{~g} / \mathrm{d}$ & 4d/sem, 1 a $3 \times 2$ a 8 rep (60\% a 95\% de 1RM) & $\begin{array}{l}\begin{array}{l}\uparrow 9,3 \% \\
* \uparrow \mathrm{RM} \text { supino } \\
* \uparrow 7 \% \text { 1RM agachamento }\end{array} \\
\end{array}$ \\
\hline Stout et al. (1999)(62) & $\begin{array}{l}24 \text { jogadores de futebol } \\
\text { americano }\end{array}$ & $\begin{array}{l}21 \mathrm{~g} / \mathrm{d} \text { por } 5 \mathrm{~d}+10,5 \mathrm{~g} / \mathrm{d} \\
\text { por } 8 \text { sem }\end{array}$ & $\begin{array}{l}\mathrm{dd} / \mathrm{sem} \\
\text { Sem } 1 \text { e } 2: 3 \times 10 \mathrm{RM} \\
\text { Sem } 3 \text { a } 6: 3 \times 5 \mathrm{RM} \\
\text { Sem } 7 \text { e } 8: 3 \times 4-3-2 \mathrm{RM} \\
\end{array}$ & $\begin{array}{l}\uparrow 3,9 \% \text { massa magra } \\
\uparrow 7,3 \% 1 \mathrm{RM} \text { supino }\end{array}$ \\
\hline Volek et al. (1999) ${ }^{(13)}$ & $\begin{array}{l}19 \text { homens treinados em } \\
\text { força }\end{array}$ & $\begin{array}{l}25 \mathrm{~g} / \mathrm{d} \text { por } 7 \mathrm{~d}+5 \mathrm{~g} / \mathrm{d} \text { por } \\
12 \mathrm{sem}\end{array}$ & $\begin{array}{l}\text { 3d/sem } \\
\text { Sem 1 e 2: } 3 \times 12 \text { RM } \\
\text { Sem 3 a 6: } 3 \times 8-10 \text { RM } \\
\text { Sem 7 a 10: } 3-4 \times 6-8 \text { RM } \\
\text { Sem 11 e 12: } 2-3 \times 3-6 \text { RM } \\
\end{array}$ & $\begin{array}{l}\text { 个6,3\%massa corporal total } \\
\uparrow 6,3 \% \text { massa magra } \\
\uparrow 24 \% \text { 1RM supino } \\
\uparrow 32 \% \text { 1RM agachamento }\end{array}$ \\
\hline $\begin{array}{l}\text { Vandenberghe et al. } \\
(1997)^{(63)}\end{array}$ & $\begin{array}{l}19 \text { mulheres saudáveis não } \\
\text { treinadas }\end{array}$ & $\begin{array}{l}20 \mathrm{~g} / \mathrm{d} \text { por } 4 \mathrm{~d}+5 \mathrm{~g} / \mathrm{d} \text { por } \\
10 \mathrm{sem}\end{array}$ & $3 \mathrm{~d} / \mathrm{sem}, 5 \times 12 \mathrm{rep}(70 \% 1 \mathrm{RM})$ & 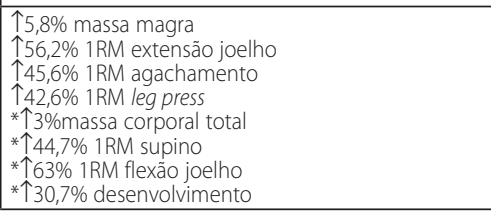 \\
\hline
\end{tabular}

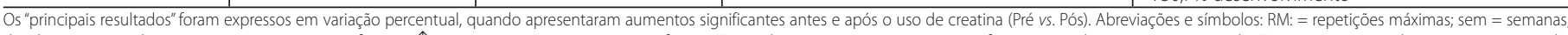

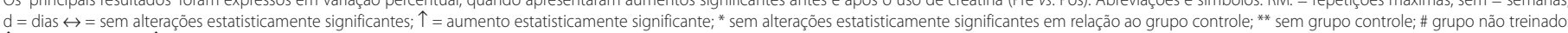
$\uparrow 7,6 \% 1$ RM supino e $\uparrow 16,1 \%$ 1RM leg press; $\S$ grupo não treinado: sem alterações significantes. 
como evidenciado nos estudos de Dangot et al. ${ }^{(38)}$, Volek et al. ${ }^{(13)}$ e Volek e Rawsom ${ }^{(39)}$. De acordo com esses estudos, a creatina possibilitaria que o sujeito desempenhasse mais repetições com a mesma carga, o que poderia se traduzir em maiores ganhos de massa magra num programa de treinamento de longo prazo. Uma grande evidência que suporta essa hipótese vem do estudo de Syrotuik et al. ${ }^{(40)}$. Esses autores relataram que sujeitos que receberam creatina, mas que desempenharam a mesma carga absoluta que o grupo placebo (apesar de serem capazes de levantar maiores cargas), apresentaram as mesmas respostas para força e hipertrofia, indicando que os benefícios advindos da creatina são associados ao aumento do volume de treinamento. Dessa forma, esses estudos sugerem que os efeitos da suplementação de creatina sobre a hipertrofia são dependentes da capacidade desse suplemento em aumentar o volume de treino.

\section{Efeitos da creatina sobre a expressão gênica e ativação das vias de trofismo muscular}

Existem recentes evidências de que a creatina pode efetivamente influenciar a transcrição gênica. Safdar et al. ${ }^{(41)}$ demonstraram que a suplementação de creatina por 10 dias é capaz de elevar a expressão de inúmeros genes envolvidos na regulação osmótica, síntese e degradação de glicogênio, remodelagem do citoesqueleto, proliferação e diferenciação de células satélites, reparo e replicação de DNA, controle da transcrição de RNA e morte celular.

Por não terem encontrado aumento agudo na síntese proteica após a suplementação de creatina em estudos anteriores ${ }^{(36,37)}$, Deldicque et al.(22) estabeleceram a hipótese de que a creatina poderia atuar em vias intracelulares que precedem os processos de síntese proteica, regulandoas em longo prazo. Os autores investigaram os efeitos desse suplemento na expressão gênica de IGF muscular (também conhecido como MGF), ativador da via PI3K-AKT/PKB-mTOR, e nas expressões gênica, proteica e fosforilada de p70s6k e 4E-BP1, efetores dessa mesma via (para detalhes dessa via e seu papel na hipertrofia, ver Zanchi et al. $\left.{ }^{(42)}\right)$. Em estudo crossover, os sujeitos foram suplementados com creatina ou placebo e submetidos a biópsias musculares no repouso, 3 e 24h após sessão de treino de força para membros inferiores. Os pesquisadores verificaram que a expressão gênica de IGF-I estava aumentada no repouso, em consequência da suplementação de creatina. Além disso, observaram aumento na fosforilação do 4E-BP1 com a suplementação, após 24h. Deldicque et al.(22), baseados no conjunto de seus dados, concluíram que a creatina pode atuar no crescimento muscular salientando o estado anabólico da célula, via IGF-I. Esse achado é corroborado por Burke et al. ${ }^{(21)}$ que verificaram aumento do conteúdo muscular de IGF-I como resultado da suplementação de creatina durante oito semanas de treinamento de força.

Além de atuar nas vias de hipertrofia, um recente estudo providenciou evidência de que a creatina pode atenuar os efeitos de corticosteroides na atrofia muscular em modelo animal ${ }^{(43)}$. Os autores relembram que os mecanismos de indução de atrofia por corticosteroides não são completamente entendidos, mas pode envolver a diminuição de expressão do IGF-I.

Baseando-se nos dados de Deldicque et al. ${ }^{(22)}$, Menezes et al. ${ }^{(43)} \mathrm{e}$ Burke et al. ${ }^{(21)}$ é possível que a creatina atue tanto salientando a hipertrofia, quanto atenuando a atrofia, já que foi demonstrado recentemente que o IGF exerce controle superior de ambas as vias tróficas (figura 1). Estudos futuros deverão investigar melhor essa possibilidade.

\section{Efeitos da creatina sobre a proliferação e diferenciação de células satélite}

Sabe-se que o núcleo da fibra muscular adulta não é capaz de sofrer mitoses, entretanto, o aumento do número de mionúcleos é necessário para a manutenção do domínio nuclear (volume de sarcoplasma pelo qual um mionúcleo é responsável pela síntese proteica) durante o processo de hipertrofia. Há diversos estudos afirmando que as células

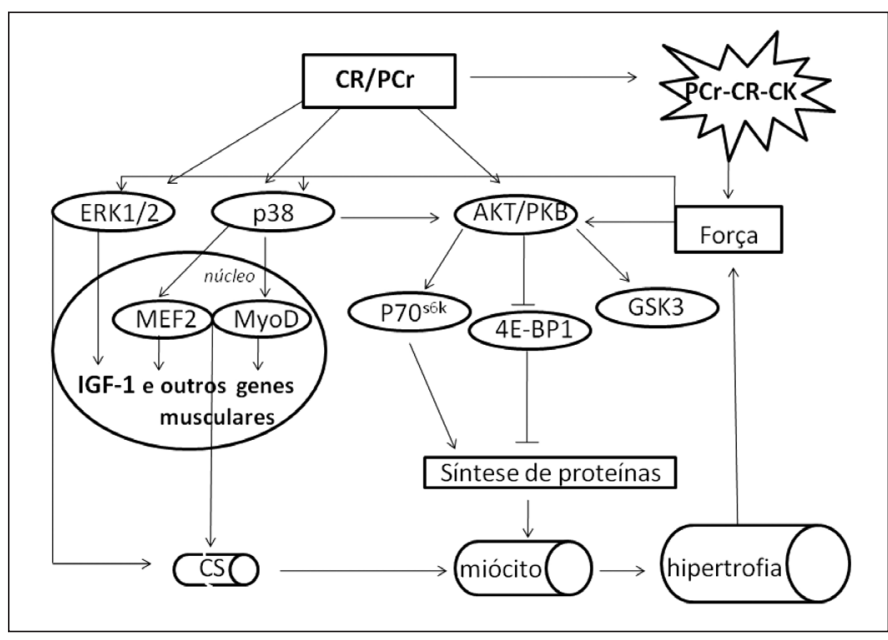

Figura 1. Mecanismos moleculares que supostamente explicariam os efeitos da suplementação de creatina na força e hipertrofia. A suplementação de creatina pode aumentar a expressão/fosforilação de genes envolvidos diretamente na síntese de proteínas e, consequentemente, hipertrofia, como IGF1-AKT/PKB e seus efetores p70 56k, GSK3 e 4-EBP1. Além disso, a creatina, via p38 e ERK1/2, pode aumentar a expressão de fatores de transcrição (MEF2 e MyoD) capazes de regular a ativação e diferenciação de células satélites (CS), necessárias para o processo hipertrófico. Paralelamente, a suplementação de creatina sabidamente salienta a provisão de energia em atividades de alta intensidade e curta duração, como o treino de força. Um aumento no volume de treino pode resultar em maiores ganhos de força e massa muscular em longo prazo. Para detalhes, consultar o sub-tópico "Efeitos da suplementação de creatina na hipertrofia". Baseado nas referências 18, 23, 64 e 65.

satélites (células quiescentes capazes de se diferenciar em fibras adultas) são as responsáveis por doarem seus mionúcleos à fibra muscular, mantendo o domínio nuclear e, assim, possibilitando a continuidade do processo hipertrófico ${ }^{(23,38,44,45)}$.

Willoughby e Rosene ${ }^{(19)}$ demonstraram que o treinamento de força combinado à suplementação de creatina é capaz de aumentar a expressão de fatores miogênicos regulatórios (da família MRFs, do inglês myogenic regulatory factors), como MRF4 e miogenina, parcialmente responsáveis pela proliferação e diferenciação de células satélites. Hespel et al. ${ }^{(33)}$ também relataram aumentos na expressão da MRF4 em indivíduos suplementados com creatina, embora não tenham encontrado alterações na expressão de miogenina.

Vierck et al. ${ }^{(46)}$ observaram aumento na atividade mitótica das células satélites de ovelhas suplementadas com creatina. Dangott et al. ${ }^{(38)}$ analisaram o efeito da suplementação de creatina sobre a hipertrofia muscular e a atividade mitótica das células satélites de ratos durante o processo de hipertrofia compensatória no músculo plantar de ratos (induzida pela remoção cirúrgica dos músculos sóleo e gastrocnêmio) e encontraram maior atividade mitótica no grupo suplementado. Olsen et al. ${ }^{(23)}$ verificaram aumento na área de secção transversa, no número de células satélites e mionúcleos em sujeitos suplementados com creatina e submetidos a treinamento de força. Segundo os autores, tais achados devem-se a já discutida ativação de MRFs mediada pela creatina.

\section{PERSPECTIVAS E CONSIDERAÇÕES FINAIS}

De acordo com Lemon ${ }^{(28)}$, a grande limitação dos estudos que concluíram que a suplementação de creatina não promove hipertrofia é que a maioria deles empregou protocolos de curto prazo e sem treinamento de força. Espera-se que em um curto período de tempo surjam novos trabalhos investigando os efeitos da creatina combinados ao treinamento de força por períodos mais prolongados (três meses em diante).

Além disso, graças ao advento da biologia molecular e o consequente avanço no detalhamento das vias de trofismo muscular, acredita-se que os mecanismos biomoleculares pelos quais a creatina exerça seu efeito ergogênico sejam bem descritos muito em breve.

Por fim, os consistentes dados presentes na literatura acerca do efeito ergogênico da creatina em atletas começaram, recentemente, a incenti- 
var o uso terapêutico dessa substância em doenças caracterizadas por acometimentos musculares. De fato, alguns trabalhos já têm demonstrado melhoras clínicas e fisiológicas decorrentes desse suplemento em pacientes com miopatias inflamatórias ${ }^{(47)}$ e distrofias musculares ${ }^{(48)}$. Uma excelente revisão sistemática sobre o tema foi recentemente publicada por Kley et al. ${ }^{(49)}$. Certamente, os efeitos terapêuticos da creatina emergem como um futuro e promissor campo de estudo.

Em conjunto, os estudos descritos nessa revisão sugerem que os ganhos de força e massa magra advindos da suplementação de creatina são consequências dos aumentos de retenção hídrica, expressão gênica e eficiência da tradução de proteínas relacionadas à hipertrofia, além da proliferação e ativação de células satélites. À luz da presente literatura, ainda não se pode afirmar com clareza se essas adaptações são oca-

\section{REFERÊNCIAS}

1. Walker JB. Creatine: Biosynthesis, Regulation and Function. Advan Enzymol 1979;50:177-242.

2. Terjung RL, Clarkson P, Eichner ER, Greenhaff PL, Hespel PJ, Israel RG, et al. The physiological and health effects of oral creatine supplementation. Med Sci Sports Exerc 2000;32:706-17.

3. Harris $\mathrm{R}$, Soderlund $\mathrm{K}$, Hultman E. Elevation of creatine in resting and exercised muscle of normal subjects by creatine supplementation. Clin Sci 1992:83:367-74

4. Stone MH, Sanborn K, Smith LL, O'Bryant HS, Hoke T, Utter AC, et al. Effects of in-season (5 weeks) creatine and pyruvate supplementation on anaerobic performance and body composition in American football players. In J Sport Nutr 1999;9:146-65.

5. Jones AM, Atter T, Georg KP. Oral creatine supplementation improves multiple sprint performance in elite icehockey players. J Sports Med Phys Fitness 1999;39:189-96.

6. Mero AA, Keskinen KL, Malvela MT, Sallinen JM. Combined creatine and sodium bicarbonate supplementation enhances interval swimming. I Strength Cond Res 2004;18:306-10.

7. Volek JS, Kraemer WJ, Bush JA, Boetes M, Incledon T, Clark KL, et al. Creatine supplementation enhances muscular performance during high-intensity resistance exercise. J Am Diet Assoc 1997;97:765-70.

8. Preen D, Dawson B, Goodman C, Lawrence S, Beilby J, Ching S. Effect of creatine loading on long-term sprint exercise performance and metabolism. Med Sci Sports Exerc 2001;33:814-21.

9. Mujika I, Padilla S, Ibanez J, Izquierdo M, Gorostiaga E. Creatine supplementation and sprint performance in soccer players. Med Sci Sports Exerc 2000;32:518-25.

10. Branch JD. Effect of creatine supplementation on body composition and performance: a meta-analysis. Int J Sport Nutr Exerc Metab 2003;13:198-26

11. Nissen SL, Sharp RL. Effect of dietary supplements on lean mass and strength gains with resistance exercise: a meta-analisys. J Appl Physiol 2003;94:651-7.

12. Jowko E, Ostaszewski P, Jank M, Sacharuk J, Zieniewicz A, Wilczak J, et al. Creatine and B-hydroxy-B-methylbutyrate (HMB) additively increase lean body mass and muscle strength during a weight-training program. Nutrition 2001:17:558-566.

13. Volek JS, Duncan ND, Mazzetti SA, Staron RS, Putukian M, Gomez AL, et al. Performance and muscle fiber adaptations to creatine supplementation and heavy resistance training. Med Sci Sports Exerc 1999;31:1147-56.

14. Tarnopolsky MA, Nutritional consideration in the aging athlete. Clin J Sport Med 2008:18:531-8.

15. Kerksick C, Harvey T, Stout J, Campbell B, Wilborn C, Kreider R, et al. International Society of Sports Nutrition position stand: Nutrient timing. J Int Soc Sports Nutr 2008:5:17.

16. Hespel P, Derave W. Ergogenic effects of creatine in sports and rehabilitation. Subcell Biochem 2007:46:245-59.

17. Aagard P. Making muscles "stronger": exercise, nutrition, drugs. J Musculoskelet Neuronal Interact 2004;4:165-74

18. Willoughby DS, Rosene J. Effectes of oral creatine and resistance training on myogenic regulatory factor expres sion. Med Sci Sports Exerc 2003;35:923-9.

19. Willoughby DS, Rosene J. Effects of oral creatine and resistance training on myosin heavy chain expression. Med Sci Sports Exerc 2001;33:1674-81.

20. Kreider RB, Almada AL, Antonio J, Broeder C, Earnest C, Greenwood M, et al. ISSN exercise \& sport nutrition review: research and recommendations. Sport Nutr Rev J 2004;1:1-44

21. Burke DG, Candow DG, Chilibeck PD, MacNeil LG, Roy BD, Tarnopolsky MA, et al. Effect of creatine supplementation and resistance-exercise training on muscle insulin-like growth factor in young adults. Int I Sport Nutr Exerc Metab 2008;18:389-98.

22. Deldicque L, Louis M, Nielens H, Dhoux M, Thissen JP, Rennie MJ, et al. Increased IGF mRNA in human skeletal muscle after creatine supplementation. Med Sci Sports Exerc 2005;37:731-6.

23. Olsen S, Aagaard P, Kadi F, Tufekovic G, Verney J, Olesen JL, et al. Creatine supplementation augments the in crease in satellite cell and myonuclei number in human skeletal muscle induced by strength training. J Physio 2006;573:525-34

24. Carvalho T, Rodrigues T, Meyer F, Lancha Junior AH, De rose EH. Modificações dietéticas, reposição hídrica, Suplementos alimentares e drogas: comprovação de ação ergogênica e potenciais riscos para a saúde. Rev Bras Med Esporte 2003;9:43-56

25. Kilduff LP, Vidakovic P, Cooney G, Twycross-Lewis R, Amuna P, Parker M, et al. Effects of creatine on isometric benchpress performance in resistance-trained humans. Med Sci Sports Exerc 2002;34:1176-83.

26. Bembem MG, Tuttle TD, Bembem DA, Knehans AW. Effects of creatine supplementation on isometric force-time curve characteristics. Med Sci Sports Exerc 2001;33:1876-81.

27. Gillian JD, Hohzorn C, Martin D, Trimble MH. Effect of oral creatine supplementation on isokinetic torque production. Med Sci Sports Exerc 2000:32:993-6.

28. Lemon PWR. Dietary Creatine Supplementation and Exercise Performance:Why Inconsistent Results? Can J App Physiol 2002;27:663-80.

29. Gualano B, Benatti FB, Ferreira JCB, Franchini E, Brum PC, Lancha Junior AH. Efeitos da suplementação de creatina no exercício intermitente de alta intensidade: divergências e recomendaçöes metodológicas. Rev Bras Cineantropom Desempenho Hum 2008;10:189-96.

30. Tarnopolsky MA, Parise G, Yardley NJ, Ballantyne CS, Olatunji S, Phillips SM. Creatine- dextrose and protein-dextrose induce similar strength gains during training. Med Sci Sports Exerc 2001;33:2044-52.

31. Volek JS, Duncan ND, Mezzetti SA, Staron RS, Putukian M, Gómez AL, et al. Performance and muscle fiber adaptations to creatine supplementation and heavy resistance training. Med Sci Sports Exerc 1999;31:1147-56.

32. Arciero PJ, Hannibal NS, Nindl BC, Gentile CL, Hamed J, Vukovich MD. Comparison of creatine ingestion and resistance training on energy expenditure and limb blood flow. Metabolism 2001;50:1429-535.

33. Hespel PB, t'Eijnde M, Van Leemputte B, Ursot PL, Greenhaff V, Labarque S, et al. Oral creatine supplementation facilitates de rehabilitation of disuse atrophy and alters the expression of muscle myogenic factors in humans. J Physiol 2001:536:625-33. sionadas por efeitos diretos da suplementação de creatina ou se são mediadas pelo aumento no volume de treinamento. Contudo, os efeitos da suplementação de creatina na promoção de ganho de massa magra e força são contundentes. Segundo Diretriz publicada pela Sociedade Brasileira de Medicina do Esporte em 2003(24), não havia evidências científicas suficientes que embasassem o emprego da suplementação de creatina com fins ergogênicos. Diante da consistência dos achados apresentados nesta revisão, acreditamos que as recomendações sobre esse suplemento precisam ser revisadas e atualizadas.

Todos os autores declararam não haver qualquer potencial conflito de interesses referente a este artigo.

34. Berneis K, Ninnis R, Häussinger D, Keller U. Effects of hyper and hypoosmolality on whole body protein and glucose kinetics in humans. Am J Physiol 1999:276:188-95.

35. Parise G, Mihic S, MacLennan D, Yarasheski K, Tarnopolsky M. Effects of acute creatine monohydrate supplementation on leucine kinetics and mixed muscle protein synthesis. J Appl Physiol 2001;91:1041-7.

36. Louis M, Poortmans JR, Francaux M, Hultman E, Berre J, Boisseau N, et al. Creatine supplementation has no effec on human muscle protein turnover at rest in the postabsorptive or fed states. Am J Physiol Endocrinol Metab 2003;284:764-70

37. Louis M, Poortmans JR, Francaux M, Berré J, Boisseau N, Brassine E, et al. No effect of creatine supplementation on human myofibrillar and sarcoplasmic protein synthesis after resistance exercise. Am J Physiol Endocrinol Metab 2003;285:1089-94.

38. Dangot B, Schultz E, Mozdziak PE. Dietary creatine monohydrate supplementation increases satellite cells mitotic activity during compensatory hypertrophy. Int J Sports Med 2000;21:13-6.

39. Volek JS, Rawson E. Scientific basis and practical aspects of creatine supplementation for athletes. Nutrition 2004;20:609-14.

40. Syrotuik DG, Bell GJ, Burnham R, Sim LL, Calvert RA, McLean IM. Absolute and relative strength performance following creatine monohydrate supplementation combined with periodized resistance training. J Strength Cond Res 2000;14:182

41. Safdar A, Nicholas JY, Snow R, Melo S, Tarnopolsky MA. Global and targeted gene expression and protein content in skeletal muscle of young men following short-term creatine monohydrate supplementation. Physiol Genomics 2008:32:219-28

42. Zanchi NE, Lancha AH Jr. Mechanical stimuli of skeletal muscle: implications on mTOR/p70s6k and protein synthesis. Eur J Appl Physiol 2008;102:253-63.

43. Menezes $L G$, Sobreira C, Neder L, Rodriques-Júnior AL, Martinez JA. Creatine supplementation attenuates corticosteroid-induced muscle wasting and impairment of exercise performance in rats. J Apply Physiol 2007;102:698-703.

44. Allen DL, Roy RR, Edgerton VR. Myonuclear domains in muscle adaptation and disease. Muscle Nerve 1999;22:1350-60

45. Kadi F, Schjerling $P$, Andersen $L L$, Charifi N, Madsen JL, Christensen $L R$, et al. The effects of heavy resistance training and detraining on satellite cells in human skeletal muscles. J Physiol 2004;558:1005-12.

46. Vierck JL, Icenoggle DL, Bucci L, Dodson MV. The effects of ergogenic compounds on myogenic satellite cells. Med Sci Sports Exerc 2003;35:769-76.

47. Chung YL, Alexanderson H, Pipitone N, Morrison C, Dastmalchi M, Ståhl-Hallengren C, et al. Creatine supplements in patients with idiopathic inflammatory myopathies who are clinically weak after conventional pharmacologic treatment: Six-month, double-blind, randomized, placebo-controlled trial. Arthritis Rheum 2007;57:694-702.

48. Tarnopolsky MA, Mahoney DJ, Vajsar J, Rodriguez C, Doherty TJ, Roy BD, et al. Creatine monohydrate enhances strength and body composition in Duchenne muscular dystrophy. Neurology 2004;62:1771-7.

49. Kley RA, Vorgerd M, Tarnopolsky MA. Creatine for treating muscle disorders. Cochrane Database Syst Rev 2007;24:CD004760

50. Bemben MG, Bemben DA, Loftiss DD, Knehans AW. Creatine supplementation during resistance training in college football athletes. Med Sci Sports Exerc 2001;33:1667-73.

51. Bermon S, Venembre P, Sachet C, Valour S, Dolisi C. Effects of creatine monohydrate ingestion in sedentary and weight-trained older adults. Acta Physiol Scand. 1998;164:147-55.

52. Burke DG, Chilibeck PD, Parise G, Candow DG, Mahoney D, Tarnopolsky M. Effect of Creatine and Weight Training on Muscle Creatine and Performance in Vegetarians. Med Sci Sports Exerc 2003;35:1946-55,

53. Candow DG, Little JP, Chilibeck PD, Abeysekara S, Zello GA, Kazachkov M, et al. Low-Dose Creatine Combined with Protein during Resistance Training in Older Men. Med Sci Sports Exerc 2008;40:1645-52.

54. Chrusch MJ, Chilibeck PD, Chad KE, Davison KS, Burke DG. Creatine supplementation combined with resistance traintng in oider men. Med Sci Sports Exerc 2001;33:2111-7.

55. Cribb PJ, Hayes A. Effects os supplement timing and resistance exercise on skeletal muscle hypertrophy. Med Sc Sports Exerc 2006;38:1918-25.

56. Cribb PJ, Williams AD, Hayes A. A creatine-protein-carboihydrate supplement enhances responses to resistance training. Med Sci Sports Exerc 2007;39:1960-8.

57. Cribb PJ, Williams AD, Stathis CG, Carey MF, Hayes A. Effects of whey isolate, creatine and resistance training on muscle hypertrophy. Med Sci Sports Exerc 2007;39:298-307.

58. Hass $\mathrm{CJ}$, Collins MA, Juncos JL. Resistance training with creatine monohydrate improves upper-body strength in patients with Parkinson disease: a randomized trial. Neurorehabil Neural Repair 2007:21:107-15.

59. Hoffman J, Ratamess N, Kang J, Mangine G, Faigenbaum A, Stout J. Effect of creatine and beta-alanine supplementation on performance and endocrine responses in strength/power athletes. Int I Sport Nutr Exerc Metab 2006;16:430-46

60. Kerksick CM, Rasmussen C, Lancaster S, Starks M, Smith P, Melton C, et al. Impact of differing protein sources and a creatine containing nutritional formula after 12 weeks of resistance training. Nutrition 2007:23:647-56.

61. Kreider RB, Ferreira M, Wilson M, Grindstaff P, Plisk S, Reinardy J, et al. Effects of creatine supplementation on body composition, strength, and sprint performance. Med Sci Sports Exerc 1998;30:73-82.

62. Stout J, Eckerson J, Noonan D, Moore G, Cullen D. Effects of 8 weeks of creatine supplementation on exercise performance and fat-free weight in football players during training. Nutrition Research 1999;19:217-25.

63. Vandenberghe K, Goris M, Van Hecke P, Van Leemputte M, Vangerven L, Hespel P. Long-term creatine intake is beneficial top muscle performance during resistance training. J Appl Physiol 1997;83:2055-63.

64. Deldicque L, Theisen D, Bertrand L, Hespel P, Hue L, Francaux M. Creatine enhances differentiation of myogenic C2C12 cells by activating both p38 and Akt/PKB pathways. Am J Physiol Cell Physiol. 2007;293:1263-71.

65. Deldicque L, Atherton P, Patel R, Theisen D, Nielens $H$, Rennie MJ, et al. Effects of resistance exercise with and without cre atine supplementation on gene expression and cell signaling human skeletal muscle. J Appl Physiol 2008;104:371-8 